\title{
Es \\ La biopolítica de los sufrimientos psíquicos
}

\author{
María Fernanda VÁsquez Valencia
}

\section{Locos y degenerados. Una genealogía de la psiquiatría ampliada}

Sandra Caponi

Lugar Editorial

Buenos Aires, 2015, 192 págs.

\section{Loucos e degenerados. Uma genealogia da psiquiatria ampliada}

Sandra Caponi

Editora Fiocruz

Rio de Janeiro, 2012, 210 págs.

En el año 2012, la editorial Fiocruz publicó la versión original en portugués del libro Loucos e degenerados. Uma genealogia da psiquiatria ampliada (reimpresa en 2014) de la investigadora y filósofa Sandra Caponi, versión que fue nominada en 2013 al premio Jabuti - uno de los más relevantes y tradicionales premios del libro en Brasil - en la categoría "psicología e psicoanálisis". Esta obra que ha ido adquiriendo gran reconocimiento entre profesionales e investigadores latinoamericanos, especialmente brasileños (cf. Rebelo, 2013; Santos, 2013; Martínez, 2013), dedicados al estudio de la historia de las ciencias, la medicina, la salud mental y la psiquiatría, ahora puede ser consultada en idioma español, gracias a la reciente edición de Lugar Editorial de Argentina. Y será esa edición en español que aquí se citará y referirá.

Durante los años 1960 y 1970, comienza a desarrollarse una serie de críticas sobre la forma cómo la psiquiatría ejerce su poder en las poblaciones. Esas críticas provienen no solo de investigadores de las ciencias sociales y humanas, sino también de algunos psiquiatras, muchos de ellos interesados en evidenciar el internamiento forzado y las pésimas condiciones de vida de las personas diagnosticadas como enfermos mentales, así como la falta de validez del diagnóstico psiquiátrico (cf. Goffman, 1974; Foucault, 1961, 1963; Cooper, 1968; Laing, 1961; Basaglia, 1987; Rosen, 1974; Szasz, 1973). A las críticas dirigidas a las instituciones manicomiales, la lucha por la desinstitucionalización del tratamiento de las alteraciones mentales, la medicalización forzada y la crítica al modelo médico, se suman un conjunto de cambios sociopolíticos, 
tales como el establecimiento del neoliberalismo, la decadencia del totalitarismo soviético, las dictaduras latinoamericanas, conformando el contexto bajo el cual se estableció un movimiento, que algunos autores prefieren denominar como "anti-psiquiátrico" (cf. Perez, 2012; Roca, 2011).

Sin embargo, la desmanicomialización, que se extendió por decenas de países, en parte gracias a la lucha de dicho movimiento iniciado en Inglaterra, Italia y Estados Unidos, y que significó cambios importantes, en términos jurídicos y políticos, en algunos planos de salud de ciertos países, también permitió el aumento de la medicalización y la hegemonía de las empresas farmacéuticas, como mediadores de una nueva estrategia neoliberal que redujo la problemática a la administración masiva de medicamentos.

En Locos y degenerados, Caponi muestra que lejos de una reflexión crítica frente a los postulados cuestionados por los defensores de los derechos de las personas diagnosticadas como enfermos mentales, representados muchas veces por el movimiento anti-psiquiátrico, ocurrió una radicalización y un retorno a las viejas tesis de la psiquiatría biológica del siglo xIx (cf. p. 63).

Ese retorno, afirma la autora, puede ser explicado, entre otras razones, por la recuperación de la hegemonía de la psiquiatría biológica, representada por los denominados neokrapelinianos, quienes, durante los años 1970, consiguen imponer una visión determinista, biologicista y neurológica de las enfermedades mentales, que se conserva hasta hoy y que influenció decididamente la edición del Manual diagnóstico y estadístico de los trastornos mentales (DSM), concretamente a partir del tercer DSM. Sumado a ese proceso disciplinar, el acelerado desarrollo de los psicofármacos, la idea de que las enfermedades mentales son producidas por un "desequilibrio químico" en el cerebro; el avance de los estudios genéticos, de las nuevas tecnologías hechas para "detectar" y "evidenciar" variaciones y cambios en el cerebro, así como los estudios estadísticos, aseguró el retorno de las tesis de la psiquiatría del siglo xIx, reforzando todavía más el reduccionismo biológico sobre las enfermedades mentales.

Ese retorno, como bien muestra Caponi en su libro, habla de la persistencia, continuidad y discontinuidad de un proceso histórico que extiende sus raíces hasta la segunda mitad del siglo xix y que comienza, como afirma la autora, siguiendo la propuesta de Michel Foucault, con el Tratado de las degenerescencias de Benedict Augusto Morel publicado en 1857. La consolidación y transformación de la teoría de la degeneración marca la emergencia de lo "no patológico" y, al mismo tiempo, la expansión del poder psiquiátrico como dispositivo de defensa de la sociedad (cf. Foucault, 2001).

A partir de Locos y degenerados es posible observar cómo la articulación de mecanismos disciplinares y reguladores que involucran la creación de una teoría, es decir, la teoría de la degeneración, el nacimiento como disciplina científica y médica de la 
psiquiatría, la relación de esta disciplina con la justicia y la definición de un conjunto de dispositivos de internación, clasificación y regulación, posibilitaron la emergencia del discurso sobre el anormal.

Desde 1857 el saber psiquiátrico se reorganiza, amplía sus fronteras más allá de lo estrictamente asilar hasta convertirse en una estrategia biopolítica encargada de "resolver" gran parte de los problemas sociales. Ese mecanismo de expansión, se debió, entre otros motivos, a la transformación de su objetivo de cura en un objetivo orientado a la prevención, así como a la constitución de una red institucional que pudiera establecer interconexiones con diferentes instancias de poder.

La conformación de esa psiquiatría ampliada involucra una transformación epistemológica del concepto de degeneración, como lo muestra Caponi a lo largo de su libro, que persiste en la psiquiatría contemporánea, travestido y transfigurado bajo otras categorías psiquiatrías como las constituciones psíquicas o las predisposiciones hereditarias.

A través de Locos y degenerados es posible también entender como la degeneración se convierte en un programa de investigación, que no solo fue fructífero en Francia, sino también en Alemania, Italia, España y América Latina. Y, al mismo tiempo, cómo ese programa agregó todo un conjunto de profesionales, desde médicos, neurólogos, psiquiatras, pasando por abogados, jueces, criminólogos, higienistas, sociólogos, hasta políticos, periodistas y literatos. La degeneración estuvo relacionada con una serie de problemas diversos, tales como la purificación de la raza, la mistura racial, el progreso de las naciones, la eugenesia, la criminalidad, la pedagogía y muchos otros temas relativos a la higiene pública y social.

En su libro, Caponi realiza un interesante estudio histórico-epistemológico, en el que analiza las condiciones de posibilidad y de emergencia de esa psiquiatría ampliada como un mecanismo biopolítico que, a partir de la segunda mitad del siglo xIx, permitió que el saber psiquiátrico expandiera sus fronteras al estudio e intervención de todas aquellas conductas, desvíos, anomalías, vicios y sufrimientos psíquicos que podían representar situaciones de conflicto o de desorden social. En la constitución de esa psiquiatría ampliada, el concepto de degeneración y la teoría de Morel tuvieron un papel central en la configuración de un marco explicativo que permitió intervenir sobre todo tipo de comportamientos categorizados como desviados.

Analizando la psiquiatría contemporánea, Caponi realiza un recorrido histórico que evidencia las permanencias y discontinuidades de ese concepto a lo largo de la configuración de la psiquiatría como disciplina científica hasta los actuales manuales de diagnóstico (DSM) hechos por la American Psychiatric Association (APA). En su opinión, los últimos años muestran una tendencia en las sociedades modernas a pensar todos los conflictos o dificultades en términos médicos y, más precisamente, en tér- 
minos psiquiátricos. Paradigma que es reforzado por el aumento, cada día más alarmante, de nuevos diagnósticos que convierten situaciones y comportamientos de la vida cotidiana, como la tristeza, la preocupación, la pérdida del apetito, los problemas familiares o laborales, la pérdida de un ser querido, en síntomas o en patologías psiquiátricas, multiplicando hasta el infinito el campo de injerencia del poder psiquiátrico. Según Caponi, la proliferación de diagnósticos psiquiátricos se legitima con la publicación del tercer Manual de diagnóstico de trastornos mentales (DSM III), en 1980. De hecho, en la última edición de ese manual (DSM v) en 2013, se incluyen 300 tipos de diagnóstico, siendo el luto y la rebeldía caracterizados como nuevos disturbios emocionales. La rebeldía, especialmente adolescente, es denominada ahora como "trastorno de oposición desafiante" (cf. Chacón, 2012). El determinismo biológico y hereditario que sirve de base para justificar la prevención y la intervención terapéutica descontextualiza y oscurece los problemas concretos que están relacionados con ese tipo de comportamientos o de situaciones cotidianas. Por eso, como afirma acertadamente Caponi, asistimos a una minimización de nuestra capacidad de reflexionar sobre nosotros mismos y ese determinismo restringe las posibilidades de crear estrategias más efectivas para dar respuesta a nuestros propios problemas (p. 16). Es precisamente ese presente problemático que hace pertinente el estudio histórico de las condiciones de posibilidad que permitieron que la psiquiatría se transformara en una estrategia biopolítica de defensa de la sociedad, teniendo como tarea principal administrar e anticipar las múltiples formas de comportamiento que pueden, tarde o temprano, afectar el orden social.

La importancia del estudio de Sandra Caponi es tanto metodológica como teórica. En primer lugar porque integra en un mismo análisis la historia conceptual de Georges Canguilhem y la genealogía de Michel Foucault, tomando de ambos elementos que permiten comprender las condiciones de emergencia y de posibilidad de la psiquiatría ampliada. Además, el libro ofrece un panorama completo del proceso a través del cual el concepto de degeneración y la teoría de Morel van sufriendo una serie de transformaciones, pero también de permanencias, evidentes en las teorías psiquiátricas que suceden los trabajos de Morel, concretamente los estudios de Valentin Magnan e Emil Kraepelin.

A lo largo de los seis capítulos Caponi desarrolla su propuesta teórica y metodológica para dar cuenta del contexto histórico de constitución de esa biopolítica de la población y de la administración de los sufrimientos psíquicos, enmarcada en una medicina de lo no patológico. En el primer capítulo titulado "Del tratamiento moral a la psiquiatría ampliada", analiza la manera como se sucede el desplazamiento de una comprensión de la locura como representación, como fenómeno histórico e social, hacia la constitución de un discurso psiquiátrico entendido como dispositivo de poder 
y de saber. Dicho análisis es hecho a partir de una lectura integrada y profunda de cuatro libros de Michel Foucault (Historia de la locura en la época clásica; Vigilar y castigar; El nacimiento de la clínica; El poder psiquiátrico) a través de los cuales Caponi realiza una doble argumentación. Por un lado, muestra cómo se da, en la obra del autor francés, ese desplazamiento así como las discontinuidades y rupturas en los argumentos que ese autor utiliza para comprender las condiciones de emergencia y de posibilidad de la psiquiatría ampliada. Analizar cómo Foucault reorganiza sus argumentos a partir de una nueva perspectiva metodológica y teórica, que integra el problema de la relación entre el poder y el saber y ya no solamente el asunto de la locura como representación o percepción social, permite visibilizar la manera cómo se establecen y circulan relaciones de poder dentro del saber médico psiquiátrico. Entender cómo se articula el saber, la verdad y el poder, en relación con ese fenómeno social e histórico llamado locura, abre toda una constelación de nuevos y variados problemas relativos al poder disciplinar, la normalización y las estrategias y tácticas de control al interior de la institución asilar y el saber médico psiquiátrico.

A partir de esa lectura, Caponi se adentra en la psiquiatría clásica, analizando especialmente los estudios de Pinel y Esquirol, para mostrar cómo ese tipo de psiquiatría se aleja de la medicina y la clínica anatomopatológica y usa el interrogatorio psiquiátrico como dispositivo disciplinar y el "cuerpo ampliado" de la familia como un cuerpo fantasmagórico a partir del cual es posible ubicar un conjunto de conflictos y patologías, así como los antecedentes individuales de la enfermedad. Para Caponi, el interés por ese cuerpo ampliado comienza en la psiquiatría clásica, pues es a través de los interrogatorios que involucran un conocimiento sobre los antecedentes familiares, a partir de los cuales es posible comprender la trama de redes causales que operan en cada caso concreto, para determinar la variedad y la especie de alienación y su tratamiento. El interrogatorio evidencia entonces la existencia de elementos que desencadenan el proceso patológico al interior de las relaciones familiares (p. 44).

En ese mismo capítulo muestra cómo el tratamiento moral, definido por Pinel como la única forma de restablecer la salud de los alienados, puede ser entendido como un dispositivo disciplinar orientado a la disciplinarización de los cuerpos y a la vigilancia continua al interior del asilo. Según la autora, no es que la psiquiatría sea una disciplina en la que el cuerpo está ausente, como argumentaba Foucault; para ella, el cuerpo juega un papel decisivo en esa red, no solamente porque desde sus inicios la psiquiatría estuvo preocupada por encontrar correlaciones entre enfermedades mentales y lesiones, sino también porque el cuerpo se localiza en el centro de la terapéutica asilar, por lo menos en el caso de Pinel, y a partir de la segunda mitad del siglo xix, será la herencia mórbida la encargada de localizar las patologías mentales en el cuerpo, particularmente en el cerebro. A través del recorrido histórico que la autora realiza en el 
primer capítulo es posible comprender que la ausencia de ese cuerpo de la anatomopatología, el diagnóstico binario (loco, no loco), la descripción de superficie de los síntomas, la clasificación de las enfermedades tomando como modelo la clasificación botánica de Lineo y el proceso de cura vinculado con la restitución de conductas y valores morales "permiten definir el marco peculiar por el cual el poder psiquiátrico ingresa en la sociedad disciplinar" (p. 49).

"Clima, cerebro y degeneración en Cabanis" es el título que recibe el segundo capítulo del libro. Analizando específicamente la obra Rapports du physique et du moral de l'homme (1802) del ideólogo francés Georges Cabanis, Caponi muestra cómo antes de que Gall publicase su estudio sobre anatomía y patología del sistema nervioso, en Cabanis ya existía un interés por articular lo físico y lo moral, atribuyendo al cerebro y al sistema nervioso un papel central en la definición de la moralidad y de las conductas humanas.

Las ideas localizacionistas de Cabanis y el modelo explicativo que generó sirvieron de punto de partida, como muestra la autora, para que los teóricos de la degeneración pudieran, a partir de la segunda mitad del siglo xIx, explicar las enfermedades mentales como alteraciones de las funciones o lesiones localizadas en el cerebro. En términos teóricos, el estudio de Cabanis permite comprender cómo el concepto de degeneración, que hasta ese momento era usado por la historia natural, ingresa en el campo médico. Tomando a Buffon como referencia, Cabanis analizará las modificaciones producidas en los cuerpos y en las conductas como efecto del calor y la humedad y estudiará la manera en que las alteraciones climáticas producen nuevas enfermedades y variaciones externas en los cuerpos, así como las influencias que produce sobre el cerebro. En Cabanis, la degeneración se presenta en el interior del cuerpo de los individuos, de modo que lo que degenera son los órganos, los tejidos y los fluidos corporales, explicando también la degradación de las facultades físicas y morales. Según Caponi, Cabanis inaugura una concepción médica de la moralidad, posibilitando también que se desarrolle un discurso optimista sobre las posibilidades de perfeccionamiento del género humano. De ese modo, si es posible pensar el mejoramiento animal y vegetal, lo es también el de los seres humanos. La regeneración como un plan de mejoramiento de las condiciones morales y físicas de los individuos y de los grupos aparece en ese horizonte discursivo entrelazado a las medidas higiénicas que permiten alterar los efectos nocivos del clima y modificar los comportamientos y los cuerpos humanos (cf. p. 75).

A través del segundo capítulo Caponi muestra la manera en que Cabanis, al relacionar la teoría de los humores, las influencias climáticas y la anatomopatología, permite el desarrollo de un conjunto de reflexiones y de estrategias de intervención sobre los cuerpos, en las que el cuerpo es comprendido a través de la integración de lo físico, 
lo moral y lo intelectual y, al mismo tiempo, un discurso que imagina el mal funcionamiento del cuerpo, especialmente del cerebro, como explicación de nuestros actos. En consecuencia, la regeneración moral de los individuos y de las razas a través de la mediación de la higiene y de la educación se convierte poco a poco en un esperanzador medio de intervención y en referencia privilegiada para la resolución de diversos conflictos sociales y morales.

En el tercer capítulo denominado "Benedict August Morel y la emergencia de la teoría de la degeneración”, Caponi analiza las condiciones de emergencia de la psiquiatría ampliada a través de la instauración, uso y consolidación de la teoría de la degeneración de Morel durante la segunda mitad del siglo xIx. Esa teoría establece una clasificación etiológica de las enfermedades mentales, que permite definir grupos y familias de degenerados, entendidos como una desviación mórbida del tipo normal de la humanidad. A pesar de que, en Morel, los procesos de degeneración son siempre el resultado de diversas influencias mórbidas tanto físicas como morales, existe un elemento que se destaca entre otros como la causa general e integradora, esto es, la transmisión hereditaria. A diferencia de las teorías clásicas (Pinel, Esquirol) en las cuales la transmisión hereditaria actuaba como una causa más, y en las cuales lo que se heredaba eran patologías semejantes, por herencia similar, en la teoría de Morel, las causas predisponentes y las causas determinantes se combinan de tal manera que pueden llegar a producir en los descendientes enfermedades distintas a las de sus progenitores y aún pueden llegar a agravarse. Ese tipo de transmisión conocida como herencia disimilar o de transformación fue clave, junto con la referencia a las lesiones cerebrales, para que el saber psiquiátrico ampliase sus fronteras más allá del ámbito asilar. La combinación de esas causas permitía crear categorías de individuos cujas semejanzas externas servían de indicadores para los diferentes tipos de degeneración, creando así los conocidos estigmas de degeneración, marcas, o signos que se presentaban en los individuos, en los grupos, que podían agravarse y ser transmitidos hereditariamente. Esa combinación, muchas veces azarosa y aleatoria, definía un espacio extendido y de fronteras difusas, como lo muestra Caponi en el tercer capítulo, en el que fueron ubicadas las denominadas enfermedades mentales y, al mismo tiempo, todo un conjunto de comportamientos descritos como aberrantes, anormales, o desviados en relación con una norma biológica, social, jurídica y pedagógica.

De ese modo, en ese capítulo, Caponi analiza detalladamente la conformación de ese suelo epistemológico que posibilitó la creación y posterior consolidación de una psiquiatría ampliada, obcecada en clasificar y patologizar los más diversos comportamientos, así como el modo en que la teoría de la degeneración se ubica en ese espacio difuso en el que desaparecen las barreras taxativas entre lo normal y lo patológico. La degeneración explicará los comportamientos más graves y más leves como el resul- 
tado de causas físicas, intelectuales, sociales y morales interrelacionadas, así como su vinculación con las predisposiciones mórbidas, los estigmas de degeneración y la herencia patológica. Ese marco teórico, afirma Caponi, es fundamental para la prevención y la anticipación de la locura fuera del espacio asilar.

En el cuarto capítulo, titulado "La consolidación de un programa de investigación: Magnan y las patologías heredo-degenerativas", la autora indaga sobre la repercusión de la teoría de la degeneración de Morel en la psiquiatría francesa de finales del siglo xIx. Caponi se concentra en analizar dicha influencia en la definición de nuevos cuadros clasificatorios, en mostrar cómo a partir de Magnan la degeneración es entendida como un desequilibrio cerebral y la forma en cómo se desarrolla un programa de investigación interesado en la medicalización de los desvíos menores, de los comportamientos cotidianos que ingresan paulatinamente en el campo de saber y de intervención psiquiátrica.

Desde la segunda mitad del siglo xix e hasta 1920, la teoría de la degeneración se consolidó como el fundamento científico que legitimó la construcción de nuevas clasificaciones de patologías mentales e intervenciones médicas sobre las conductas de individuos y de grupos. La consolidación de ese programa de pesquisa se evidencia en la multiplicidad de artículos que durante esos años discuten la problemática en las principales revistas médicas francesas, Annales d'Hygiene Publique et de Mèdecine Legal y los Annales Médico-Psichologiques. A partir de los estudios de Valentin Magnan, uno de los principales discípulos de Morel, el problema de la degeneración es entendido como un desequilibrio cerebral o una desarmonía del cerebro, caracterizado por estigmas físicos, psíquicos e delirios. El desequilibrio, que sustituye la búsqueda por lesiones cerebrales específicas, permite entender la diversidad de los síntomas de los degenerados en función de la parte del cerebro afectada, los diversos cuadros patológicos que puede sufrir un individuo a lo largo de su vida, y la forma en que, a través de la herencia, diversas manifestaciones y comportamientos pueden ser transmitidos a los descendientes, evolucionando y llegando a ser irreversibles. La definición de esos cuadros sintomáticos como "síndromes de degeneración" facilita la clasificación de ese conjunto variable y diverso de comportamientos ubicados en una región difusa entre lo normal y lo patológico. Como afirma Caponi, higienistas, médicos y criminólogos se lanzan a la tarea de estudiar y describir ese amplio espectro de los "síndromes" que incluían desde manías, delirios, perversiones y demencias hasta pequeñas anomalías de comportamiento como miedos, temores y tristezas. Sobre la identificación entre las conductas cotidianas y las patologías psiquiátricas se fueron construyendo las bases epistemológicas de la psiquiatría moderna o, en otras palabras, de esa psiquiatría ampliada, que asume cada gesto, comportamiento y conducta como síntoma indicativo de un síndrome, trastorno o patología por venir. 
En el quinto capítulo, denominado "Emil Kraepelin y la persistencia de la degeneración en la psiquiatría moderna”, Caponi continua mostrando cómo las mismas preocupaciones y los mismos intereses presentes en los teóricos degeneracionistas sirven de fundamento a los estudios de Kraepelin, conocido como el padre de la psiquiatría moderna. En ese capítulo, la autora se detiene a analizar los elementos de ese programa de pesquisa que persisten en la denominada psiquiatría moderna y encuentra que, aunque de manera transfigurada, metamorfoseada y disfrazada, buena parte de las cuestiones presentes en Kraepelin era también la de los degeneracionistas. Lo que resulta interesante puesto que la psiquiatría actual se reconoce heredera directa de esa tradición científica inaugurada por Kraepelin. Realizando un delicado y detallado análisis de comparación histórica entre algunos de los trabajos del psiquiatra alemán y de Morel, Magnan y otros degeneracionistas, Caponi muestra, alejándose de ciertos análisis historiográficos que pretenden colocar a Kraepelin en un horizonte discursivo diferente al de Morel, que muchos de los argumentos y estrategias de estudio de los desvíos y de los fenómenos patológicos se mantuvieron, casi de manera intacta, en los 50 años que separan a Kraepelin de Morel: la vinculación de lesiones cerebrales con las patologías psiquiátricas y las psicosis de degeneración; la preocupación por explicar la transmisión hereditaria de patologías, comportamientos y hechos sociales; la búsqueda de una clasificación nosológica precisa; la referencia a los estudios estadísticos y de psiquiatría comparada entre países y regiones; y el uso de fichas con preguntas estandarizadas que fueron utilizadas como estrategia para fundamentar de manera objetiva las clasificaciones nosológicas y las locuras de degeneración (cf. p. 14,1). Si bien, como muestra la autora, las fichas son una novedad en el repertorio de Kraepelin, tanto él como Morel, no solamente se preocuparon por definir estrategias analíticas para clasificar enfermedades o establecer nosologías, sino que también pretendieron crear dispositivos de intervención del espacio social, capaces de anticipar, prevenir y controlar los comportamientos anormales o desviados y las patologías mentales. Esa psiquiatría preventiva, orientada a la regeneración y a la moralización de masas por medio de medidas higiénicas presente en el discurso de Morel, se agudiza en Kraepelin como una forma de legitimar intervenciones psiquiátricas en el tejido social para anticipar desvíos y conductas indeseables.

A lo largo de ese capítulo, Caponi analiza detalladamente las transformaciones, limitaciones y permanencias del concepto de degeneración en Kraepelin, también analiza la biologización de los hechos sociales y la forma en que el saber estadístico se integra a su discurso para legitimar tanto las clasificaciones como las intervenciones psiquiátricas.

Esas ideas tienen continuidad en el sexto capítulo, titulado "Herencia y degeneración: de Kraepelin a los neo-kraepelinianos”, en el que Caponi expone cómo la problemática de la degeneración forma parte de cada una de las diferentes clasificaciones 
de enfermedades mentales que aparecen en las ediciones del Manual de psiquiatría clínica de Kraepelin, texto que marcó considerablemente la psiquiatría de las primeras décadas del siglo xx, no solamente en Europa y los Estados Unidos, sino también en América Latina. El capítulo expone cómo algunas de las tesis defendidas por el psiquiatra alemán fueron retomadas y recuperadas por la psiquiatría moderna, durante la década de los años 1970, específicamente por un grupo de psiquiatras de la Universidad de Washington, los denominados neo-kraepelinianos, teniendo una influencia directa, como muestra la autora, en la reformulación de la clasificación de diagnósticos elaborada por la Asociación de Psiquiatras Americanos (APA), concretamente a partir de su tercera edición publicada en 1980. El objetivo principal del capítulo es mostrar como las bases epistemológicas de Kraepelin reaparecen en el discurso de los neo-kraepelinianos, analizando las continuidades y diferencias entre esos discursos, para comprender las razones que llevaron a ese grupo de investigadores a reconocer como válidas las premisas construidas por la psiquiatría de la segunda mitad del siglo xIx. A pesar de que los estudios de los neo-kraepelinianos parecen indicar una actualización y una continuidad en el esfuerzo por definir, a partir de los últimos descubrimientos de la neurofisiología cerebral, la estadística y la genética, clasificaciones confiables para las patologías mentales, Caponi argumenta que, en esencia, se trata de los mismos criterios de exclusión y de inclusión de la época de Kraepelin. En la opinión de la autora de Locos y degenerados, una misma matriz epistemológica persiste en todos esos discursos, marcada por un claro determinismo biológico que convierte los sufrimientos individuales, la historia de vida de los sujetos, en marcadores que pueden ser medidos y definidos patológicamente.

Aunque históricamente existen también claras diferencias entre todos esos discursos, y de que no se trata de afirmar que sean esencialmente iguales, Caponi insiste en que la psiquiatría actual continua interesada en desvendar los mismos secretos que estimularon al programa de investigación de los degeneracionistas, o sea, la relación entre las patologías psiquiátricas y la herencia, la construcción de una psiquiatría preventiva atenta al carácter evolutivo de las patologías y la búsqueda por la localización cerebral de los sufrimientos psíquicos. Caponi cierra con broche de oro su libro realizando algunas reflexiones finales en las que muestra la pertinencia del análisis histórico, epistemológico y conceptual como una forma de indagar y criticar problemas del presente.

Finalmente, esta reseña no pretende ser más que una aproximación parcial a los interesantes y provocadores planteamientos de Sandra Caponi en Locos y degenerados, por lo que invito a que los lectores se acerquen a este libro, que no solamente es una lectura obligada para quienes estudien ese tipo de temáticas, sino que también, gracias a la apasionada y amena escritura usada por la autora, resulta de gran provecho aún para aquellas personas que no estén interesadas directamente en esas problemáticas.@ 
Agradecimientos. Agradezco al programa CAPES, PEG-PG, por la concesión de la beca para realizar mis estudios de doctorado.

\author{
María Fernanda VÁsquez Valencia \\ Programa de Pós-Graduação Interdisciplinar \\ em Ciências Humanas, \\ Universidade Federal de Santa Catarina, \\ Florianópolis, Brasil. \\ mfdavasquez@gmail.com
}

\title{
The bio-politics of psychic sufferings
}

\section{REFERENGIAS BIBLIOGRÁFIGAS}

Basaglia, F. Los crímenes de la paz. México: Siglo xxi, 1987.

Chacón, P. DSM y el universo psi: diagnósticos a la medida del mercado. Revista de Cultura, 2012. Disponible en: <http://www.revistaenie.clarin.com/ideas/dsm-dsm5-psicologia-mercado-poderdiagnostico-psiquiatrico_o_664,133801.html>. Acceso en: 24, oct. 2014.

Cooper, D. The dialetic of liberation. London: Peguin Books, 1968.

Foucault, M. Histoire de la folie à l'âge classique. Paris: Plon, 1961. . Naissance de la clinique. Une archéologie du regard médical. Paris: PUF, 1963.

. Os anormais. Curso no Collège de France, 1974-1975. São Paulo: Martins Fontes, 2001.

Goffman, E. Manicômios, prisões e conventos. São Paulo: Perspectiva, 1974.

LaIng, R. The self and others. London: Tavistock Publications, 1961.

Martínez, A. Sandra Caponi, "Loucos e degenerados": uma genealogia da psiquiatria ampliada. Historia $y$ Sociedade, 25, p. 219-44, 2013.

Perez, C. Una nueva anti-psiquiatría. Críticas y conocimiento de las técnicas del poder psiquiátrico. Santiago: Lom Ediciones, 2012.

Rebelo, F. Da teoría da degeneração de Morel à classificação das doenças mentais de Kraepelin. História, Ciência e Saúde-Manguinhos, 20, 4, p. 1756-60, 2013.

Roca, A.V. Anti-psiquiatría; desconstrucción del concepto de enfermedad mental y crítica de la "razón psiquiátrica”. Nómadas, Revista Crítica de Ciencias Sociales y Jurídicas, 31, 3 p. 1-18, 2011.

Rosen, G. Locura y sociedad: sociología histórica de la enfermedad mental. Madrid: Alianza Editorial, 1974. SAntos, A. R. Loucos e degenerados: uma genealogia da psiquiatria ampliada. Cadernos de Saúde Pública, 29,1, p. 202-6, 2013.

SzAsz, T. El mito de la enfermedad mental. Buenos Aires: Amorrortu, 1973.

\section{然然}

